\title{
TBM
}

\section{Predicting organizational readiness to implement HIV pre- vention with couples using practitioners' intentions: testing a heuristic}

\author{
Susan S. Witte, ${ }^{1, \oplus}$ Rogerio Pinto, ${ }^{2}$ C. Jean Choi, ${ }^{3}$ Melanie M. Wall ${ }^{3}$
}

${ }^{1}$ Columbia University School of Social Work, 1255 Amsterdam Ave, New York, NY 10027, USA

${ }^{2}$ University of Michigan School of Social Work, 1080 S University Ave, Ann Arbor, Ml 48109, USA

${ }^{3}$ New York State Psychiatric Institute, Columbia University Medical Center, 1051 Riverside Drive, New York, NY 10032, USA

Correspondence to: Susan S.Witte, ssw12@columbia.edu

Cite this as: TBM 2020;10:155-162 doi: 10.1093/tbm/iby121

(c) Society of Behavioral Medicine 2018. All rights reserved. For permissions, please e-mail: journals.permissions@oup.com.

\section{Abstract}

Couple-based interventions may play a key role in ending the AIDS epidemic. Progress has been made in demonstrating successful implementation of both manual-based and webbased modalities of couple-based HIV prevention in clinical trials. To ensure real world implementation, however, we need a better understanding of how best to prepare organizations to support such interventions. We sought to examine which domains of staff-reported organizational readiness predicted providers' intention to deliver a couple-based HIV-prevention intervention. Organizational readiness was assessed at baseline from 253 facilitators enrolled in a randomized clinical trial testing dissemination and implementation of a couple-based HIV prevention program (2007-2012). Consistent with current organizational-readiness theory, we measured general capacities; capacities specific to a couple-based intervention; and staff motivation to implement the intervention. We used multilevel regression models to examine the influence of these capacities on intention to implement at 6-, 12-, and 18-month follow-up, adjusting for staff age, education, role, years of service, and randomized condition. Higher perceived organizational resources $(B=0.126, p=.028)$ and better staff motivation $(B=0.510$, $p=.009$ ) were significant predictors of increased intention to facilitate Connect. Higher organizational resource availability and stronger motivation to facilitate the intervention are key domains that could inform administrator and staff training to strengthen readiness for couple-based programs. However, further research is needed to clarify the role of these domains regarding actual implementation.

\section{Keywords}

Couple-based, Implementation, Organizational Readiness, HIV prevention

\section{INTRODUCTION}

Poised to end the epidemic by 2030, global AIDS researchers are calling for innovation in HIV prevention implementation efforts. We now know that couple-based interventions are more effective in promoting protected sex, HIV testing, and motherto-child prevention treatment uptake when directly compared with interventions delivered to individuals [1]. Further, recent critiques of the gap in HIV care have highlighted the need to target linkage to and retention in HIV care for individuals living with the virus in order to avert more new HIV infections $[2,3]$, and this is best accomplished by working with

\section{Implications}

Practice: HIV services organizations may not need strong intervention specific capacities in order to succeed at implementation of couple-based HIV prevention, as long as staff has the motivation to implement and perceive that organizational capacities are present.

Policy: Policy makers who wish to promote couple-based or other HIV prevention strategies may consider strengthening organizational level capacities within HIV service agencies, including provision of targeted funding to do so, as a first level of effort.

Research: Future research is needed to better delineate more specifically which organizational readiness domains influence and predict actual couple-based HIV prevention implementation.

individuals living with HIV and their main sexual partners together [1]. This evidence suggests that implementation of couple-level interventions should be a priority in efforts to end the epidemic. Successful implementation of evidence-based couple-level interventions that promote linkage to and retention in HIV care for people living with HIV, as well as other demonstrated risk reduction behaviors regardless of HIV status, should result in reducing individual and community viral load and reduced exposure to higher risk behaviors among serodiscordant couples $[4,5]$. As the locus of patient and client receipt of behavioral HIV prevention programming, leaders in community-based organizations are faced with how best to reduce barriers to implementation. Organizational facilitators of HIV prevention implementation include alignment in views regarding the potential benefit of the program, knowledge about the program, having a planned implementation approach, a desire to change agency prevention approaches, adequate staffing, and organizational stability [5]. 
In a prior study, we described the successful implementation of a couple-level intervention following training in manual or web-based modalities [6]. Little is known, however, about the specific factors of organizational readiness that may predict implementation and how to direct agencies to improve readiness for such interventions [7-9]. In this study, we examine organizational readiness for implementation of Connect, a couple-based program endorsed by the U.S. Centers for Disease Control and disseminated through the Diffusion of Evidence-Based Interventions in their high-impact prevention program [10]; and the unique considerations for organizational readiness needed for implementation of couples-based programs more broadly [6].

\section{Measuring organizational readiness}

Across multiple sectors (corporations, healthcare, government), organizational readiness, or the "extent to which organizational members are both psychologically and behaviorally prepared to implement change" [11], is a well-established predictor of program implementation [8, 11, 12]. Nilsen [12] refers to organizational readiness for change as an implementation theory in and of itself, while others describe it as one stage in the overall process. Readiness assessment provides a foundation for change throughout the implementation process and as such is critical to any effective change strategy. Readiness can be considered or measured generally, in terms of the organization, or specifically, in terms of a particular program. We have focused on practitioner readiness to implement Connect, a couple-based intervention. However, little research is available on the development of system readiness for evidence-based intervention implementation, including the steps that organizations can take to assess such readiness. Existing measures lack consistency in conceptualization and terminology-limited evidence of reliability and validity, few empirical studies of the construct, and considerable conceptual ambiguities and disagreements in terms of understanding and measuring readiness $[11,13]$. Both Weiner and Gagnon, in recent comprehensive reviews, highlight that one measure. Lehman et al.'s [14] Texas Christian University (TCU) Organizational Readiness for Change (ORC) includes both individual and organizational measures and has psychometric validity. The TCU emphasizes four areas of readiness: (1) motivation, (2) resources, (3) staff attributes, and (4) organizational climate.

In one of the most widely accessed manuscripts in the journal Implementation Science, Weiner et al. [8] argue that organizational readiness is a multilevel, multifaceted construct referring to members' shared resolve to implement change (commitment) and shared belief in collective capability to do so (efficacy). They emphasize the importance of both general and stafflevel capacities, noting a need to attend to collective commitment (associated with staff attributes) and efficacy (associated with staff motivation). Through the lens of the Interactive Systems Framework [15] and the Quality Implementation Framework [16], Scaccia et al. [17] build on the work of Weiner et al. and highlight that organizational readiness for change requires not only "general capacities" (staff perceptions of structural-level attributes) but also "innovation-specific capacities" (staff perceptions of capacity specific to the intervention, program, or new service to be implemented), as well as individual-level motivation (defined by any number of variables, including the five attributes of innovation as set forth by Rogers [18]). This conceptualization uses the formula: $R=M C^{2}$ (readiness = motivation $\times$ general capacity $\times$ intervention-specific capacity), emphasizing that motivation is not enough by itself; neither are general and specific capacities in the absence of motivation. The authors promote this as a heuristic as opposed to a theory, indicating that readiness may incorporate these variables in varying degrees, depending on the innovation or program targeted and the specific circumstances of the agency and staff.

We empirically examine whether organizational readiness for change variables consistent with these new ideas increase intention to implement a couple-level intervention among 253 providers at 80 HIV services agencies in New York State. Given the uniqueness and complexity of couple-based programs, and the skills required for a practitioner to implement such programs, we hypothesized that the intervention-specific capacities would be stronger predictors of intention to implement compared with general capacities or motivation.

\section{METHODS}

The current study used baseline and outcome data (6-, 12-, and 18-month follow-up) from an intervention trial (R01-MH080659) that examined implementation of Connect, a couple-based HIVprevention program [10, 19, 20]. Organizational readiness was assessed at baseline from the 253 participating facilitators enrolled. Consistent with current organizational-readiness theory described earlier, we measured general capacities; capacities specific to a couple-based intervention; and staff motivation to implement the intervention. The outcome, intention to implement, was measured over the three follow-up time points.

Full details for the main trial are reported elsewhere [6]. Connect is theoretically and empirically derived [21], with demonstrated efficacy in reducing risk among HIV-negative, HIV-positive, and serodiscordant heterosexual couples [22, 23] and adaptations domestically and globally with multiple high-risk 
population [24-26]. In 2009, the trial enrolled 253 staff members in 80 services agencies in New York State to determine whether training and technical assistance (TA) using a Web-based modality [27] would yield greater adoption compared with using a manual-based modality. Eligible agencies were those providing HIV services in New York State; had "notfor-profit" status in the USA; provided HIV prevention services to heterosexual men and women; and agreed to send at least one participating staff member to receive training on one of two versions of the Connect program (traditional or Multimedia). To recruit CBOS, we first identified the networks of HIV service agencies receiving state and federal funding in New York State, including Community Service Providers (CSPs) and Multi Service Agencies (MSAs). In addition, we identified and contacted 145 agencies identified through five websites representing coalitions of HIV services organizations. To our knowledge, all of the CSPs and MSAs were duplicated within the 145 agencies we independently identified. Seventyone of the 80 agencies in the final sample are among the 145 agencies that receive direct HIV prevention services funding from the NYSDOH AIDS Institute. This sample is generally representative of CBOs providing HIV prevention services in New York State. Each enrolled $\mathrm{CBO}$ was asked to identify one staff person as the "administrator" and up to five facilitators to participate. HIV services agencies are highly variable in terms of their size, scope of service, and available financial resources. Given this variability, and the relatively small sample size, we decided that a matched pair approach would best guard against imbalance across arms after random assignment. Agencies were recruited, matched on key variables, and randomly assigned to two conditions (Web-based vs. manual-based Connect) [6].

Staff gave informed consent and completed an online baseline assessment. All participants received a 4-day face-to-face training session, followed by TA calls at 2 and 4 months post-training, and completed online follow-up assessments at 6,12, and 18 months post-training. Institutional Review Board approval was secured for all study activities from the authors' institution. Implementation findings indicated that the web-based modality did not yield greater overall implementation of the intervention compared with the manual-based modality [6].

\section{MEASURES}

Demographic characteristics measured included age in years, race, and ethnicity, preferred language, level of education, sexual orientation, number of years in HIV services, and primary job role (e.g., administrator, program coordinator, case manager, peer educator).

\section{Outcome measures}

In this analysis, we did not examine implementation, but rather intention to implement, which is the strongest cognitive predictor and proxy measure for implementation [28-31]. The reason was because the parent study implementation outcomes found that-consistent with diffusion of innovations theory-there was slow movement toward actual implementation over the first 6-18 months post-training [32]. At the 6-, 12-, and 18-month follow-ups, only $2.7 \%, 3.2 \%$, and $8.2 \%$ of facilitators, respectively, reported implementing complete cycles of Connect. Implementation was increasing, but slowly. At the time of our study follow-up, the relative number was too small to provide a more meaningful outcome for the purposes of exploring predictors like readiness. This may have been due to several factors, including loss of funding at agencies due to the economic crisis of 2008-2010, or rates of uptake in general and the need for longer-term follow-up to capture implementation. Given the "low $n$ " number, we suffer in implementation science due to challenges of conducting large-scale trials and the time it takes from training to implementation over time [33]. In this study, we will examine "intention to implement." In a critical review of experimental evidence for intentions engendering behavior change, Webb and Sheeran [31] find that a medium to large change in intention engenders a small to medium change in behavior, thus intention has a significant impact on behavior, but the size of this effect is smaller than correlational tests suggest. We caution that we would not characterize our focus on intention instead of implementation as due to a failed implementation attempt. Slow uptake does not designate failure. Instead, it underscores the importance of longer term (more than 18 months) follow-up in implementation studies. We see the value in examining the utility of Scaccia et al.'s [17] organizational readiness heuristic given the uniqueness of this data set. Using intention as a proxy with strong empirical evidence for such proxy affords us the ability to examine what the experience of these 253 practitioners tell us about organizational readiness components.

Intention to implement was assessed on an 11-point Likert scale reflecting strength of belief (e.g., measured from 0 to 10,10 being strongest) in this question: "I intend to implement Connect sessions in the next six months."

\section{Predictor variables}

Consistent with the heuristic readiness model described earlier, we measured (1) general organizational capacities, (2) intervention-specific capacities, and (3) practitioner motivation to implement Connect, as follows.

General Organizational Capacities were measured using an adaptation of the TCU ORC scale. Highlighted in recent reviews as among the few organizational-readiness measures with strong psychometric properties [9, 11, 13], the ORC includes 115 items representing 18 scales covering four domains: resources, staff attributes, organizational 
climate, and motivation. Items are measured on a five-point Likert scale, ranging from strongly disagrees to strongly agree. Resources included 25 items including, for example, the adequacy of Offices (four items, e.g., "facilities here are adequate for couple counseling"), Staffing (six items, e.g., "there are enough staff here to meet current client needs"), Training Resources (four items, e.g., "this program holds regular in service training") within the agency (Cronbach alpha $=0.59$ ). Staff attributes included 20 items including, for example, Growth (five items, e.g., "you have enough opportunities to keep your counseling skills up to date"); and Efficacy (five items, e.g., "you have the skills needed to conduct effective couple counseling" (Cronbach alpha $=0.69)$. Organizational Climate included 30 items, for example, Clarity of Mission and Goals (five items, e.g., "staff understand how this program fits as part of the treatment system in your community"), Staff Cohesiveness (six items, e.g., "staff here get along well"), and Staff Autonomy (five items, e.g., "management trusts your professional judgement") (Cronbach alpha $=0.84)$. Motivation was measured at the intervention-specific level (discussed subsequently) but not from the ORC. Because review of the data indicated that some scale item values reflected poor reliability, some were dropped to strengthen reliability. The rationale, scale descriptions, and psychometric properties of the ORC are reported in detail by Lehman et al. [14].

Intervention-Specific Capacities related to the couple-based intervention were assessed on an 11-point Likert scale reflecting strength of belief (e.g., endorsement strength measured from 0 to 10 , 10 being strongest). These items were developed in consultation with our Community Collaborative Board members during study preparation and followed conceptually and theoretically measures of organizational capacities. Intervention-specific resources included three items, e.g., "The time needed to conduct CONNECT will prevent the agency from implementing CONNECT" and "The cost of conducting CONNECT will prevent the agency from implementing CONNECT" (Cronbach alpha $=0.78$ ); staff attributes included four items, e.g., "I believe that I would be able to successfully implement the CONNECT intervention/intervention" and "I would receive personal satisfaction from being able to implement CONNECT" (Cronbach alpha $=0.90)$. Climate included five items, e.g., "Our agency management will not be supportive of implementing CONNECT" and "Our agency clients are not likely to be interested in CONNECT" (Cronbach alpha $=0.58)$.

Motivation was not measured using the TCU "motivational factors." Instead, consistent with the theory and definition of motivation by Scaccia et al. [17], which defines motivation as intervention specific, we used the five items representing endorsement of innovation attributes defined by Rogers
[18], e.g., "I believe that including CONNECT in our prevention programming will improve our ability to reduce risk among heterosexual couples" and "I believe that I will see the contributions that CONNECT will make to our clients" (Cronbach alpha $=0.89)$.

\section{Data analysis}

Agency characteristics and provider sociodemographic characteristics were summarized using frequencies and means (standard deviations). Multilevel regression models of providers within services agencies were used to examine relationships between baseline general and program-specific capacities and outcomes across time, adjusting for staff age, education, primary role, years of service, and randomized condition. Such models allowed us to account for within-agency homogeneity, or providers nested within agencies, using a random effect. We tested a model that included general organizational resources, staff attributes, and organizational climate, as well as program-specific capacities and motivation to implement, consistent with the heuristic proposed by Scaccia et al. [17]. Analyses were conducted using Statistical Analysis System (SAS) 9.4 [34].

\section{RESULTS}

\section{Agency and participant characteristics}

A total of $80 \mathrm{HIV}$ services agencies participated. The mean HIV/STI-prevention budget for all agencies was $\$ 1,068,910$ ( $\mathrm{SD}=3,940,797$ ); the median was $\$ 362,500$ (range: $0-\$ 35,000,000)$. The mean number of full-time prevention staff in each agency was 18; the median was 9 (range: $0-216$ ). Of the 80 agencies, there was an average of three participating providers from each agency (range 1-7); four agencies had only one participating provider. Of the 253 participating study staff, most participants were female (71.5\%), over 40 years of age (58.1\%), and African American (41.1\%). On average, participants had $3.7(S D=1.3$ ) years of experience in HIV services and $5.4(S D=5.3)$ years of employment at their current agencies.

\section{Outcomes}

Table 1 shows the results of the multilevel regression model examining the relationship between each element of general and intervention-specific capacities and motivation on intention to implement the intervention. Higher general resources ( $B=0.126, p=.028)$, and better intervention-specific staff motivation $(B=0.510, p=.009)$ were significant predictors of increased intention to implement Connect. No other dimensions of general or intervention-specific capacities were found to be predictors of increased intention to implement. No participant demographic characteristics were found 
Table 1 | Results of the multilevel regression model predicting intention to implement connect

\begin{tabular}{|c|c|c|c|}
\hline Effect & Estimate & SE & $p$-value \\
\hline Age & 0.000027 & 0.018 & .999 \\
\hline Education (ref: High School) & & & .383 \\
\hline College/Technical & 0.624 & 0.488 & .202 \\
\hline Graduate level or higher & 0.240 & 0.514 & .641 \\
\hline Years of HIV Service (ref: Under 1 year) & & & .689 \\
\hline $1-2$ years & 1.053 & 0.777 & .176 \\
\hline $2-5$ years & 0.146 & 0.657 & .824 \\
\hline $5-10$ years & 0.298 & 0.666 & .655 \\
\hline $10+$ years & 0.214 & 0.661 & .747 \\
\hline Primary role (ref: Administrator, program director) & & & .572 \\
\hline Case manager, social worker & -0.354 & 0.466 & .448 \\
\hline Educator, counselor, trainer & 0.348 & 0.435 & .424 \\
\hline Outreach worker, peer educator & -0.012 & 0.667 & .985 \\
\hline Multimedia condition (ref: Traditional) & 0.223 & 0.408 & .586 \\
\hline Time (ref: 6 months) & & & $<.0001$ \\
\hline 12 months & -1.254 & 0.214 & $<.0001$ \\
\hline 18 months & -1.727 & 0.220 & $<.0001$ \\
\hline \multicolumn{4}{|l|}{ General Organizational Capacities } \\
\hline Resources & 0.126 & 0.057 & .028 \\
\hline Staff attributes & 0.027 & 0.052 & .605 \\
\hline Organizational climate & -0.054 & 0.050 & .283 \\
\hline \multicolumn{4}{|l|}{ Intervention-specific capacities } \\
\hline Resources & -0.183 & 0.093 & .051 \\
\hline Staff attributes & -0.069 & 0.207 & .737 \\
\hline Organizational climate & -0.076 & 0.135 & .571 \\
\hline Motivation & 0.510 & 0.195 & .009 \\
\hline
\end{tabular}

to be predictors of increased intention to implement. Overall, the average level of intention to implement significantly decreased from 6 months to 12 months follow-up by 1.3 points $(B=-1.25, p<$ $.0001)$, and from 12 months to 18 months follow-up by 0.50 points $(B=-0.50, p<.0001)$.

\section{DISCUSSION}

Findings suggest that of all general capacities, organizational resources, in addition to staff motivation, best demonstrated increases in intention to implement a couple-based intervention. Given the emphasis in the innovation literature on the lure of novelty in early adoption, we expected that elements of the intervention-specific capacities domain would have played a positive role in intention to implement this couple-based intervention-but the data suggest that they did not.

In a synthesis of the 25-year history of the DEBI program-dissemination of evidence-based interventions for HIV prevention in the USA-Collins and Sapiano [20] point to the importance of agencies deciding whether they should focus first on building general or intervention-specific capacities. They report that intervention-specific capacity building will not suffice to ready an agency for implementation if that agency also has a need for general capacity building; our findings support the need for general capacity building. Similarly, findings from a recent systematic review of organizational readiness and its relationship to innovation adoption in addiction services found that resources need to be more effectively directed to addressing organizational deficits prior to engaging in a process of innovation adoption. In other words, organizational capacity needs to come first [9].

Consistent with Scaccia et al. [17] and with Weiner [8], motivation (efficacy) was important to one's intention to implement, as was stronger perceived organizational-level resources. Inconsistent with Scaccia, however, intervention-specific resources did not predict intention. We consider a number of possible explanations for this finding.

One explanation for this finding may be that HIV services agencies' missions often combine a social-service agenda with a social change agenda. Witness, for example, that the evolution of many larger agencies began through much smaller task forces from individuals in the gay community, e.g., Gay Men's Task Force, or for individuals who inject drugs. Strong social activism by communities working in and with these agencies throughout the 1990s promoted improved access to and funding for 
antiretroviral medications. Historically, this collective social identity was formed in response to having shared experiences or having been similarly affected by HIV, making staff members at HIV services agencies highly dedicated to their clients' and communities' needs [35]. Our study took place during the domestic and global fiscal crisis beginning in 2009 when most participating agencies lost up to a third of their program funding and during a time when some HIV/AIDS advocates believe the domestic epidemic was largely ignored [36]. In the face of yet another assault on resources and community as part of a longer history of the HIV/AIDS crisis in the USA, these providers may have expressed an intention to provide services based only on their motivation and their willingness to work with whatever organizational resources are available [35].

Another explanation may be that Connect was recognized by participating providers as yet another "DEBI," an evidence-based intervention sanctioned by the Centers for Disease Control and Prevention (CDC) for implementation in a services setting. Since many participating staff already had experience with other DEBIs, there may have been a belief that they were being asked to assimilate and implement yet one more DEBI program, that despite it being a couple-based intervention, the fact that it was a packaged DEBI suggested that it was not so different than other, similar programs, and that the novelty of a couple-based intervention may not necessarily require more couple- or program-specific resources.

Another explanation may be found in another study by the authors. In an examination of provider behaviors across $36 \mathrm{HIV}$ services CBOs, we found that in those offering DEBIs, practitioners were more likely to engage in other evidence-based services, for example, active referral and linkage-making to the HIV Continuum of Care [37]. The organizational culture of offering DEBIs historically may influence intention to implement additional programming. We know that among the 80 participating agencies in the present study, most offered at least one DEBI at the time of recruitment into the study (mean and median $=1$, range was $0-7$ ). Related to this explanation is the change in HIV prevention policy domestically during the trial. In 2011, the CDC initiated the High Impact Prevention policy, which fundamentally altered the landscape of prevention implementation nationwide by shifting the target and accessibility of funding to organizations and limiting the available scope of HIV prevention interventions [37]. Awareness of the shifting policy may have influenced participant perceptions with regard to the need for intervention-specific resources to implement the couple-based DEBI.

Participant characteristics (age, education, role in the agency, and years in HIV service), which are distinct from "staff attributes" in the organizational readiness literature, did not play an important role in intention to implement. This may have been due to some level of self-selection bias where only those with relative interest in implementing Connect selected to participate. Or again, the shared mission of HIV services providers discussed earlier may explain this phenomenon as well. We are unaware of HIV prevention intervention implementation studies to date that show participant characteristics like age or education significantly influencing outcomes.

Finally, findings may reflect the limitation of using intervention-specific readiness measures at baseline in an intervention trial when participants have not yet been exposed to the intervention. Interventionspecific resources may need context to be accurately assessed. If, in the absence of an intervention training, participants were unaware of what resources might be necessary to implement the couple-based intervention, they may not be able to accurately assess this component.

Our work builds on the emerging science of readiness to implement evidence-based interventions. Our parent study suffered from slow implementation levels, adding to the "small $n$ " problem noted by Proctor et al. [33], which challenges progress in implementation science. The notion of "slow implementation levels," however, is also problematic in the sense that the field does not yet know just what "slow" means. What is an expected rate of uptake of a new program, specifically one on HIV prevention? Connect uptake was consistent with that of other DEBI programs within HIV services agencies. To address this in the current study, we used the outcome of intention to implement to overcome this problem. Weiner suggests that organizational-readiness strategies are equifinal; in other words, there is no "one best way" to increase organizational readiness for change. Scaccia et al. [17] assert that while equifinal, readiness requires at least some presence of all three elements-organizational and intervention-specific capacities and also motivation-to exist at all. Our study finds the presence of capacity in two of the three domains to be significant, leading to successful "readiness" to intend to implement.

Depending on the intervention, other general or intervention-specific capacities may be required to strengthen readiness. If Weiner's assertion is true, we cannot necessarily argue that more of any one particular element of the missing capacities might strengthen readiness. We do know, from theory and empirical evidence, that strengthening intention is a prerequisite to actual implementation. The challenge may be in understanding how much-even if only a minimal amount-of any particular element or capacity is enough to predict intention to implement, and (ultimately) actual implementation.

Implied in our findings is the importance of offering training and TA to enhance these general and intervention-specific domains-staff attributes (sense of efficacy, growth, adaptability) and organizational climate (mission clarity, agency cohesion, 
autonomy, managerial support). Additional funding and time for training is nearly always a source of need for improved programming. Champions or other administrative leaders could emphasize the increased importance of couple-based interventions given the goals of ending the epidemic by 2030 and to engage more effectively and rapidly outcomes related to linkage to care and treatment as prevention efforts. A greater investment by funders for organizations to strengthen either organizational capacity/resources and/or intervention specific resources may serve to increase intention and actual implementation of couple-based HIV prevention over time.

Because we found in this study a reduced level of intention to implement over time, we recognize the need to maintain the level of motivation and enthusiasm for intervention implementation. To respond, we strongly recommend and endorse booster training sessions or additional proactive TA sessions to sustain (or improve) levels of intention and of implementation. We also recommend partnerships between intervention researchers and practitioners to continually innovate new ways to support implementation; empirically driven structures may inspire practitioners to adopt and implement evidence-based interventions [38].

\section{Limitations}

The data are based on self-report, which can be biased or influenced by poor recall. While intention to implement is a strong proxy for implementation in theory, practical implications of findings based on intention are difficult to estimate. Further, the findings may have been influenced by measurement. The challenges of measuring organizational readiness have been well documented [8, 9, 13]. Minor modifications were made to the TCU ORC subscales for use with HIV providers. Some subscale items were dropped due to multicollinearity and poor alpha levels. We believe, however, that these changes reflect better the organizational context of HIV services programs and that adapted measures are more relevant. We used new "intervention-specific" measures constructed by investigators consistent with the literature and which yielded strong Cronbach's alphas. Constructs in these domains overlap; they are not always discrete. This makes it more difficult to discern if they are truly "general," "intervention-specific," or "motivation" capacities. Future work is needed to examine these more clearly. Further, this study sought to test a model of organizational readiness empirically using data from this trial. We sought to specifically examine the role of organizational readiness components in implementation of a couple-based intervention within HIV services agencies. We did not set out to examine organizational readiness as one of many explanatory factors for implementation; hence we cannot estimate the relative influence readiness may have compared with other factors.

\section{CONCLUSIONS}

Practitioners' access to training and resources supporting more innovative and unique aspects of programming, including a couple-based intervention, may not be essential for implementation as long as general resources and motivation are available. This may be good news for agencies interested in the innovation of promoting couple-based programs, or it may be a finding specific to the history and experience of HIV/AIDS services programs in New York City. Future studies of couple-based program implementation should examine the role of each element of these capacities as they influence intention to implement and actual implementation, as well as the ways in which they may vary but still succeed in predicting intention or implementation.

Acknowledgments: We wish to acknowledge all participating agencies and staff, as well as their clients, whose time and effort made this study possible.

\section{Compliance with Ethical Standards}

Funding: This study was funded by R01-MH080659 to PI Witte.

Conflict of interest: The authors declare that they have no conflicts of interest.

Authors' Contributions: SSW conceived of and developed the manuscript with input from RMP. MW and JC conducted data analysis. All authors contributed to writing, reviewing and finalizing the manuscript.

Ethical Approval: All procedures performed in studies involving human participants were in accordance with the ethical standards of the institutional and/or national research committee and with the 1964 Helsinki declaration and its later amendments or comparable ethical standards. This article does not contain any studies with animals performed by any of the authors.

Informed Consent: Informed consent was obtained from all individual participants included in the study.

\section{References}

1. Crepaz N, Tungol-Ashmon MV, Vosburgh HW, Baack BN, Mullins MM. Are couple-based interventions more effective than interventions delivered to individuals in promoting HIV protective behaviors? A meta-analysis. AIDS Care. 2015;27(11):1361-1366.

2. Shah M, Risher K, Berry SA, Dowdy DW. The epidemiologic and economic impact of improving HIV testing, linkage, and retention in care in the United States. Clin Infect Dis. 2016;62(2):220-229.

3. Bartlett JG. The conspicuous gap between HIV care and HIV public health. Medscape J Med. 2016. Available at http://www.medscape.com/ viewarticle/864796. Accessibility verified November 20, 2018.

4. El-Bassel N, Gilbert L, Witte S, Wu E, Hunt T, Remien RH. Couple-based HIV prevention in the United States: advantages, gaps, and future directions. J Acquir Immune Defic Syndr (1999). 2010;55(Suppl 2):S98S101. doi:10.1186/s13012-015-0216-2

5. Kegeles SM, Rebchook G, Tebbetts S, Arnold E; TRIP Team. Facilitators and barriers to effective scale-up of an evidence-based multilevel HIV prevention intervention. Implement Sci. 2015;10(1):50. doi:10.1186/ s13012-015-0216-2

6. Witte SS, Wu E, El-Bassel N, et al. Implementation of a couple-based HIV prevention program: a cluster randomized trial comparing manual versus Web-based approaches. Implement Sci. 2014;9(1):116. doi:10.1186/ s13012-014-0116-x 
7. Chilenski SM, Olson JR, Schulte JA, Perkins DF, Spoth R. A multi-level examination of how the organizational context relates to readiness to implement prevention and evidence-based programming in community settings. Eval Program Plann. 2015;48:63-74. doi:10.1016/j. evalprogplan.2014.10.004

8. Weiner B. A theory of organizational readiness for change. Implement Sci. 2009;4(1):67. doi:10.1186/1748-5908-4-67

9. Kelly P, Hegarty J, Barry J, Dyer KR, Horgan A. A systematic review of the relationship between staff perceptions of organizational readiness to change and the process of innovation adoption in substance misuse treatment programs. J Subst Abuse Treat. 2017;80:6-25. doi:10.1016/j.jsat.2017.06.001

10. CONNECT. Available at http://www.effectiveinterventions.org/en/ HighlmpactPrevention/Interventions/Connect.aspx. Accessibility verified February 10, 2016.

11. Weiner BJ, Amick H, Lee SY. Conceptualization and measurement of organizational readiness for change: a review of the literature in health services research and other fields. Med Care Res Rev. 2008;65(4):379-436.

12. Nilsen P. Making sense of implementation theories, models and frameworks. Implement Sci. 2015;10(1):53. doi:10.1186/ s13012-015-0242-0

13. Gagnon MP, Attieh R, Ghandour el K, et al. A systematic review of instruments to assess organizational readiness for knowledge translation in health care. Plos One. 2014;9(12):e114338.

14. Lehman WE, Greener JM, Simpson DD. Assessing organizational readiness for change. J Subst Abuse Treat. 2002;22(4):197-209.

15. Wandersman A, Duffy J, Flaspohler P, et al. Bridging the gap between prevention research and practice: the interactive systems framework for dissemination and implementation. Am J Commun Psychol. 2008;41(3-4):171-181.

16. Meyers DC, Durlak JA, Wandersman A. The quality implementation framework: a synthesis of critical steps in the implementation process. Am J Commun Psychol. 2012;50(3-4):462-480.

17. Scaccia JP, Cook BS, Lamont A, et al. A practical implementation science heuristic for organizational readiness: $\mathrm{R}=\mathrm{MC2}$. J Commun Psychol. 2015;43(4):484-501.

18. Rogers EM. Diffusion of Innovations. New York, NY: Simon and Schuster; 2010.

19. Collins CB Jr, Wilson KM. CDC's dissemination of evidence-based behavioral HIV prevention interventions. Transl Behav Med. 2011;1(2):203-204.

20. Collins CB Jr, Sapiano TN. Lessons learned from dissemination of evidence-based interventions for HIV prevention. Am J Prev Med. 2016;51 (4 Suppl 2):S140-S147.

21. Sormanti M, Pereira L, El-Bassel N, Witte S, Gilbert L. The role of community consultants in designing an HIV prevention intervention. AIDS Educ Prev. 2001;13(4):311-328.

22. El-Bassel N, Witte SS, Gilbert L, et al. The efficacy of a relationship-based HIV/STD prevention program for heterosexual couples. Am J Public Health. 2003;93(6):963-969.

23. Witte SS, El-Bassel N, Gilbert L, Wu E, Chang M, Steinglass P. Recruitment of minority women and their main sexual partners in an HIV/STI prevention trial. J Womens Health (Larchmt). 2004;13(10):1137-1147.
24. Wu E, El-Bassel N, McVinney LD, et al. Feasibility and promise of a couple-based HIV/STI preventive intervention for methamphetamine-using, black men who have sex with men. AIDS Behav. 2011;15(8):1745-1754.

25. El-Bassel N, Jemmott JB, Landis JR, et al.; NIMH Multisite HIV/STD Prevention Trial for African American Couples Group. National Institute of Mental Health Multisite Eban HIV/STD prevention intervention for African American HIV serodiscordant couples: a cluster randomized trial. Arch Intern Med. 2010;170(17):1594-1601.

26. Pettifor A, MacPhail C, Nguyen N, Rosenberg M, Parker L, Sibeko J. Feasibility and acceptability of project connect: a couples-based HIV-risk reduction intervention among young couples in Johannesburg, South Africa. AIDS Care. 2014;26(4):476-482.

27. Moretti F, Witte S. Using new media to improve learning: multimedia connect for HIV/AIDS risk reduction and the triangle initiative. In: Toward Equity in Health: a New Global Approach to Health Disparities. New York: Springer Publishing Company; 2007.

28. Fishbein M, Triandis H, Kanfer F, Becker M, Middlestadt S. Factors influencing behavior and behavior change. In: Baum A, Revenson TA, Singe JE eds. Handbook of health psychology. Mahwah, NJ: Lawrence Erlbaum Associates Publishers; 2001. pp. 1-17.

29. Michie S, Johnston M, Abraham C, Lawton R, Parker D, Walker A; "Psychological Theory" Group. Making psychological theory useful for implementing evidence based practice: a consensus approach. Qual Saf Health Care. 2005;14(1):26-33.

30. Armitage $\mathrm{CJ}$, Conner M. Efficacy of the theory of planned behaviour: a meta-analytic review. Br J Soc Psychol. 2001;40(Pt 4):471-499.

31. Webb TL, Sheeran P. Does changing behavioral intentions engender behavior change? A meta-analysis of the experimental evidence. Psycho Bull. 2006;132(2):249-268.

32. Compendium of evidence-based behavioral interventions. Available at http://www.cdc.gov/hiv/prevention/research/compendium/rr/index.html. Accessibility verified February 10, 2016.

33. Proctor EK, Landsverk J, Aarons G, Chambers D, Glisson C, Mittman B. Implementation research in mental health services: an emerging science with conceptual, methodological, and training challenges. Adm Policy Ment Health. 2009;36(1):24-34.

34. SAS. Base SAS 9.4 Procedures Guide: Statistical Procedures. 5th ed Cary, NC: SAS Institute; 2017.

35. Miller RL. Innovation in HIV prevention: organizational and intervention characteristics affecting program adoption. Am J Community Psychol. 2001;29(4):621-647.

36. The Black AIDS Institute. 30-Year AIDS report card: which presidents make the grade. In 30-Year AIDS Report Card: Which Presidents Make the Grade. 2011. Available at https://blackaids.org/blog/30-year-aidsreport-card-which-presidents-make-the-grade. Accessibility verified November 20, 2018

37. Pinto RM, Witte SS, Filippone PL, Choi CJ, Wall M. Policy interventions shaping hiv prevention: providers' active role in the HIV continuum of care. Health Educ Behav. 2018;45(5):714-722.

38. Pinto RM, Wall MM, Spector AY. Modeling the structure of partnership between researchers and front-line service providers: strengthening collaborative public health research. I Mix Method Res. 2014;8(1):83-106. 